\title{
HALO EXPANSION IN COSMOLOGICAL HYDRO SIMULATIONS: TOWARD A BARYONIC SOLUTION OF THE CUSP/CORE PROBLEM IN MASSIVE SPIRALS
}

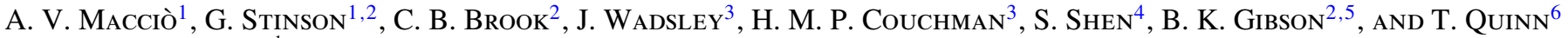 \\ ${ }^{1}$ Max-Planck-Institut für Astronomie, 69117 Heidelberg, Germany; maccio@mpia.de, stinson@mpia.de \\ ${ }^{2}$ University of Central Lancashire, Jeremiah Horrocks Institute for Astrophysics \& Supercomputing, Preston PR1 2HE, UK \\ ${ }^{3}$ Department of Physics and Astronomy, McMaster University, Hamilton, Ontario, L8S 4M1, Canada \\ ${ }^{4}$ Department of Astronomy and Astrophysics, University of California Santa Cruz, Santa Cruz, CA 95064, USA \\ ${ }^{5}$ Department of Astronomy \& Physics, Saint Mary's University, Halifax, Nova Scotia, B3H 3C3, Canada \\ ${ }^{6}$ Astronomy Department, University of Washington, Seattle, WA 98195-1580, USA \\ Received 2011 September 26; accepted 2011 November 21; published 2011 December 9
}

\begin{abstract}
A clear prediction of the cold dark matter (CDM) model is the existence of cuspy dark matter halo density profiles on all mass scales. This is not in agreement with the observed rotation curves of spiral galaxies, challenging on small scales the otherwise successful CDM paradigm. In this work we employ high-resolution cosmological hydrodynamical simulations to study the effects of dissipative processes on the inner distribution of dark matter in Milky Way like objects $\left(M \approx 10^{12} M_{\odot}\right)$. Our simulations include supernova feedback, and the effects of the radiation pressure of massive stars before they explode as supernovae. The increased stellar feedback results in the expansion of the dark matter halo instead of contraction with respect to $N$-body simulations. Baryons are able to erase the dark matter cuspy distribution, creating a flat, cored, dark matter density profile in the central several kiloparsecs of a massive Milky-Way-like halo. The profile is well fit by a Burkert profile, with fitting parameters consistent with the observations. In addition, we obtain flat rotation curves as well as extended, exponential stellar disk profiles. While the stellar disk we obtain is still partially too thick to resemble the Milky Way thin disk, this pilot study shows that there is enough energy available in the baryonic component to alter the dark matter distribution even in massive disk galaxies, providing a possible solution to the long-standing problem of cusps versus cores.
\end{abstract}

Key words: cosmology: theory - galaxies: structure - hydrodynamics - methods: numerical

Online-only material: color figures

\section{INTRODUCTION}

The theory of cold dark matter (CDM) provides a successful framework for understanding structure formation in the universe (e.g., Spergel et al. 2003; Komatsu et al. 2011). In this paradigm, dark matter first collapses into small halos, which merge to form progressively larger halos. Galaxies are thought to form out of gas which cools and collapses to the centers of these dark matter halos (White \& Rees 1978). Properties of dark matter halos have been extensively studied via collisionless $\mathrm{N}$-body simulations of the growth of primordial fluctuations into gravitationally bound structures. Numerical simulations have facilitated detailed predictions for a wide range of properties of dark matter halos at all epochs (e.g., Macciò et al. 2008; Prada et al. 2011).

Dissipationless cosmological simulations have also raised problems for the CDM scenario on small scales, one of which is the central slope of the dark matter density profile of virialized objects. $N$-body simulations predict a central concentration, with a logarithmic slope of $\approx-1$ (Navarro et al. 1997; Diemand et al. 2005; Springel et al. 2008). Such a "cuspy" matter distribution is not supported by observations of the rotation curves of spiral galaxies, which have revealed that the dark halos encompassing disk galaxies have a constant density core (e.g., Salucci \& Burkert 2000; Oh et al. 2008; Spano et al. 2008; Kuzio de Naray et al. 2009). Donato et al. (2009) have recently analyzed the rotation curves of a large sample of galaxies ranging over all Hubble types and with luminosity as high as $M_{B} \approx-22$. Their analysis shows that the central surface density is nearly constant and independent of galaxy luminosity. This issue presents a major challenge for the otherwise successful CDM cosmological model.

By construction, dissipationless simulations do not include baryons. While on large scales, the effect of gas and stars can be neglected, this is not true on small scales, where baryons can be gravitationally dominant. For this purpose, cosmological hydrodynamical simulations have been extensively used to directly address the question of galaxy properties in the CDM scenario (e.g., Navarro \& Steinmetz 2000; Abadi et al. 2003; Brook et al. 2004; Robertson et al. 2004; Okamoto et al. 2005; Macciò et al. 2006; Governato et al. 2007; Sánchez-Blázquez et al. 2009; Scannapieco et al. 2009; Stinson et al. 2010; Piontek \& Steinmetz 2011; Agertz et al. 2011).

The response of dark matter to baryonic infall (and star formation) is still highly debated. During galaxy formation, as cosmic gas cools and condenses toward the halo center and forms stars, dark matter particles are pulled inward and increase their central density. This process is dubbed "adiabatic contraction" (e.g., Blumenthal et al. 1986; Gnedin et al. 2004). Halo contraction is present in the vast majority of cosmological hydrodynamical simulations (see Gnedin et al. 2011 and references therein), yet these simulated galaxies fail to reproduce observed rotation curves due to their too centrally concentrated stellar and dark matter profiles. In order to reproduce observational data, like the rotation-velocity-luminosity and size-luminosity relations, models with NO adiabatic contraction (Gnedin et al. 2007; Courteau et al. 2007) or even with expansion (Dutton et al. 2007, 2011) are required (but also see Trujillo-Gomez et al. 2011).

To resolve these discrepancies, theoretical arguments and simulations have proposed baryonic processes that can result 
in producing an expansion of the dark matter halo. Gas bulk motions, possibly supernova-induced in regions of high star formation activity, and the subsequent energy loss of gas clouds due to dynamical friction can transfer energy to the central dark matter component (Navarro et al. 1996; Mo \& Mao 2004; El-Zant et al. 2001; Ogiya \& Mori 2011).

Mashchenko et al. $(2006,2008)$ have pointed out that there is another (possibly more relevant) effect, namely, the gas bulk motion can induce substantial gravitational potential fluctuations and a subsequent reduction in the central dark matter density. Recently Governato et al. (2010) have presented a highresolution cosmological simulation of a dwarf galaxy that was able to create a cored dark matter profile at $z=0$ within the CDM scenario and to reproduce several properties of observed dwarf galaxies. Resolution of an inhomogeneous interstellar medium (ISM), and strong supernova-driven outflows, which inhibits the formation of bulges, resulted in the decrease of the dark matter density to less than half of what it would otherwise be within the central kiloparsec of these low-mass objects $\left(M_{\mathrm{dm}} \approx 10^{10} M_{\odot}\right)$. In a further analysis of this simulation, Pontzen \& Governato (2011) showed that the flattening was the result of relatively small starbursts in the center of the protogalaxy, which contribute over many cycles to a gradual transfer of energy from the baryons to the dark matter. This mechanism is closely related to matter outflows, but does not require violent, sudden mass loss. In a recent paper Brook et al. (2011) showed that large fractions of the gas that is expelled from the central regions of galaxies returns via a large-scale galactic fountain to form stars at later times: this greatly increases the occurrence of outflows from the inner regions for a galaxy of given stellar mass, and thus any flattening mechanism that relates to outflows will be significantly enhanced.

Dekel \& Silk (1986) showed that supernovae can eject matter from halos up to $100 \mathrm{~km} \mathrm{~s}^{-1}$, but it has yet to be seen what impact this might have on dark matter profiles, or how the addition of radiation pressure feedback might change things. So, while observations show evidence for flattened dark matter density profiles up to $\mathrm{L}^{*}$ galaxies, the question remains whether there is enough energy input from baryons in more massive objects in order for these processes to be effective in altering the dark matter density profile of spiral galaxies with a dark matter mass of the order of $10^{11}-10^{12}$.

In this Letter, we present a high-resolution cosmological hydrodynamic simulation of a massive spiral galaxy that includes cooling due to hydrogen and heavier "metal" elements (Shen et al. 2010), UV background radiation (Haardt \& Madau from CLOUDY), a simple commonly used star formation prescription, adiabatic supernova feedback (Stinson et al. 2006), along with feedback from the early radiation produced by massive stars (G. Stinson et al. 2011, in preparation; see Brook et al. 2011). Our results show that reasonable baryonic feedback is able to create a density core in the dark matter distribution even for massive spiral galaxies approaching the mass of our own Milky Way.

This Letter is organized as follows: in Section 2 we describe our simulations, in Section 3 we present our results on the dark matter radial density profile, and finally, Section 4 is devoted to conclusions and discussion.

\section{SIMULATIONS}

In this Letter we use g5664, a cosmological zoom simulation drawn from the McMaster Unbiased Galaxy Simulations (MUGS). See Stinson et al. 2010 for a complete description of the creation of the initial conditions. g5664 has a total mass of $7 \times 10^{11} M_{\odot}$, a spin parameter of 0.024 , and a last major merger at $z=3.4$. Inside $r_{\text {vir }}$ at $z=0$, there are $4 \times 10^{5}$ dark matter particles with a mass of $1.1 \times 10^{6} M_{\odot}$, a similar number gas particles with mass $2.1 \times 10^{5} M_{\odot}$ and between $3.5 \times 10^{5}$ and $10^{6}$ stars with mass $5.5 \times 10^{5} M_{\odot}$ depending on the star formation and feedback recipes used. Using the physics employed in the original MUGS simulations, g5664 formed an exponential disk with an exponential bulge with a central surface brightness of $\mu_{i}=18$, had a total face-on magnitude of $M_{r}=-21.7$ and $g-r$ color of 0.52 . In many ways, g5664 is a similar initial condition to the Guedes et al. (2011) eris simulation, except eight times lower resolution. We will refer to this simulation as the low feedback run (LFR).

The new version of g5664 was again evolved using the smoothed particle hydrodynamics code GASOLINE (Wadsley et al. 2004). For this high feedback run (HFR), three changes were made which resulted in a stronger implementation of stellar feedback: (1) the Kroupa et al. (1993) initial mass function (IMF) used in MUGS was changed to the more commonly used Chabrier (2003) IMF which creates more massive stars and hence more energy per stellar mass created. (2) We use a star formation density threshold of $n_{\text {th }} 9.3 \mathrm{~cm}^{-3}$ and an efficiency of $c_{\star}=0.1$. (3) The new runs assume the energy input from supernovae is $10^{51} \mathrm{erg}$ instead of the $4 \times 10^{50} \mathrm{erg}$ used in MUGS. (4) The new run includes radiation released by the massive young stars before they explode as supernovae. All of these changes are based on a parameter search that will be reported in G. Stinson et al. (2011, in preparation). It will be shown that each of these changes is necessary to produce more realistic galaxies as described in Section 3. For details on the implementation of the radiative feedback, see Brook et al. (2011), though the work presented here deposits $17.5 \%$ of the massive star luminosity as thermal energy instead of the $10 \%$ reported there. Again, this is motivated by the production of a more realistic galaxy. We note here that the energy deposition from supernovae and radiation pressure couple inefficiently to the gas. Both types of feedback are deposited into the high-density gas found in the disk where the cooling time is shorter than the dynamical timescales resolved in the simulation.

We emphasize that our feedback scheme relies on subgrid physics. It is not possible to fully resolve the sites where star formation happens and energy is fed back into the ISM. Our simple description of the radiation pressure mechanism is a relatively crude initial representation. Finally, we also ran a dark-matter-only version of g5664 ( $N$-body run), with the same particle resolution.

\section{RESULTS}

In this Letter we focus on the effects of feedback on the dark matter distribution in our simulated galaxy. A more comprehensive study of the properties of simulated galaxies using such a strong feedback implementation will be presented in a forthcoming series of papers. In brief, the simulation presented here does a much better job reproducing the observed properties of galaxies including a flatter rotation curve, an exponential surface brightness profile, and a stellar mass, $6 \times 10^{9} M_{\odot}$, in better agreement with what halo abundance matching predicts a $7 \times 10^{11} M_{\odot}$ should contain (Moster et al. 2010). This latter point is important, as it indicates that the large-scale outflows inherent in this study may be necessary.

Figure 1 shows the dark matter density profile in our three simulations. The pure dark matter run ( $N$-body, black line) 


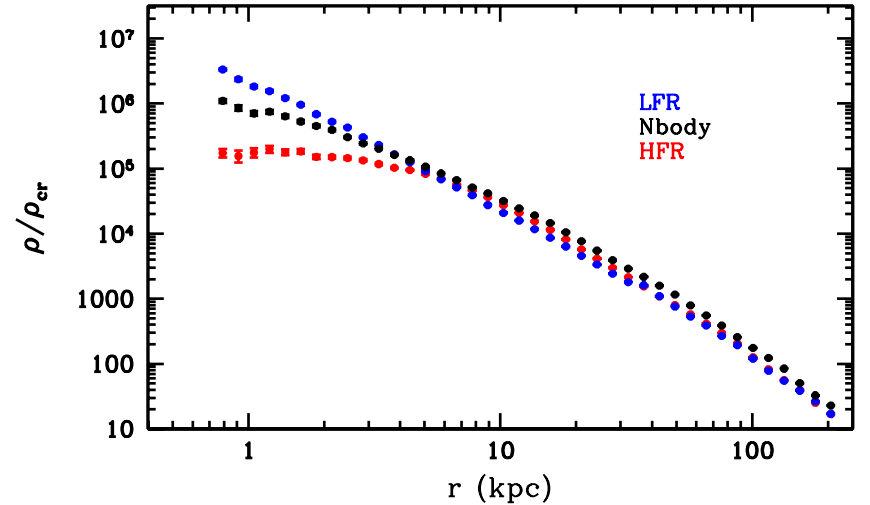

Figure 1. Density profile for only the dark matter in the three different realizations of our Galaxy. The blue line shows the low feedback run (LFR), the black line shows the dark matter only run ( $N$-body), and the red line shows the higher feedback case. The blue curve shows evidence for adiabatic contraction, the black one presents the usual NFW profiles, while the red one clearly shows a cored profile, in agreement with observations.

(A color version of this figure is available in the online journal.)

is well fit by a Navarro-Frenk-White (NFW) profile with concentration parameter of $c=6$, in agreement with cosmological expectations (Muñoz-Cuartas et al. 2011). The profile of the LFR (dotted-blue) shows evidence of significant adiabatic contraction, with dark matter pulled toward the inner regions by the centrally concentrated baryons. The inner profile is fit with a single power law $\left(\rho \propto r^{-\alpha}\right)$, with $\alpha=2$. As reported in Stinson et al. (2010), this dark matter peak is accompanied by a high concentration of baryonic material at the center of the galaxy, represented by a centrally peaked rotation curve and a high bulge-to-total ratio. None of these features agree with observations, which do not support the adiabatic contraction scenario at these mass scales. The lowest curve is our HFR, which uses a Chabrier IMF and radiation pressure feedback. The dark matter density profile follows the pure dark matter run in to $r \approx 5 \mathrm{kpc}$, but then it notably flattens to clearly reveal the presence of a core in the inner region.

The dark matter density profile of the HFR can be fit with a Burkert profile (Burkert 1995):

$$
\rho(r)=\frac{\rho_{0} r^{3}}{\left(r+r_{0}\right)\left(r^{2}+r_{0}^{2}\right)} .
$$

This profile, when combined with appropriate baryonic gaseous and stellar components, is found to reproduce very well the observed kinematics of disk systems (e.g., Salucci \& Burkert 2000; Gentile et al. 2007). The two free parameters $\left(\rho_{0} ; r_{0}\right)$ can be determined through a $\chi^{2}$ minimization fitting procedure: in our case this led to $\rho_{0} / \rho_{c r}=1.565 \times 10^{5}$ and $r_{0}=9.11 \mathrm{kpc}$. The simulated dark matter profile with its Burkert fit is shown in the upper panel of Figure 2.

Results of Donato et al. (2009), showing the central surface density $\mu_{0}$, defined as the product of the halo core radius and central density $\left(\mu_{0} \equiv r_{0} \rho_{0}\right)$ of galactic dark matter halos, are shown (open circles) in the lower panel of Figure 2. Our simulated galaxy is overplotted as a red star. Not only can the simulation be fit with a Burkert profile, but the cored profile of g5664 HFR agrees with observed density profiles. The magenta squares are lower mass simulations which have similar high feedback prescriptions as the HFR, with slight calibration changes as these simulations have eight times better resolution. Detailed properties of these simulations will be presented in a forthcoming paper (C. B. Brook et al. 2011, in preparation).
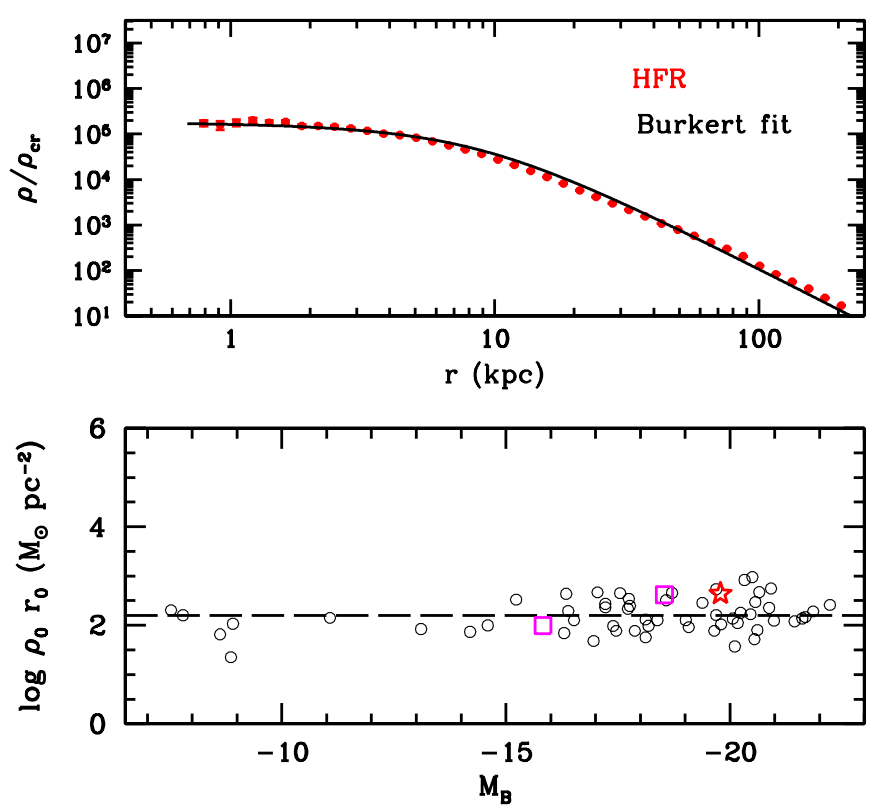

Figure 2. Upper panel: density profile of the dark matter component in g5664 HFR, and fitting Burkert profile with a core size of $r_{0}=9.11 \mathrm{kpc}$. Lower panel: the relation between luminosity and dark matter halo surface density. Open symbols represent observational results, while our simulated galaxy is represented by the red star. The dashed line is the fit to this relation, suggested by Donato et al. (2009). The magenta squares are lower mass simulations which have similar high feedback prescriptions as the HFR (C. B. Brook et al. 2011, in preparation).

(A color version of this figure is available in the online journal.)

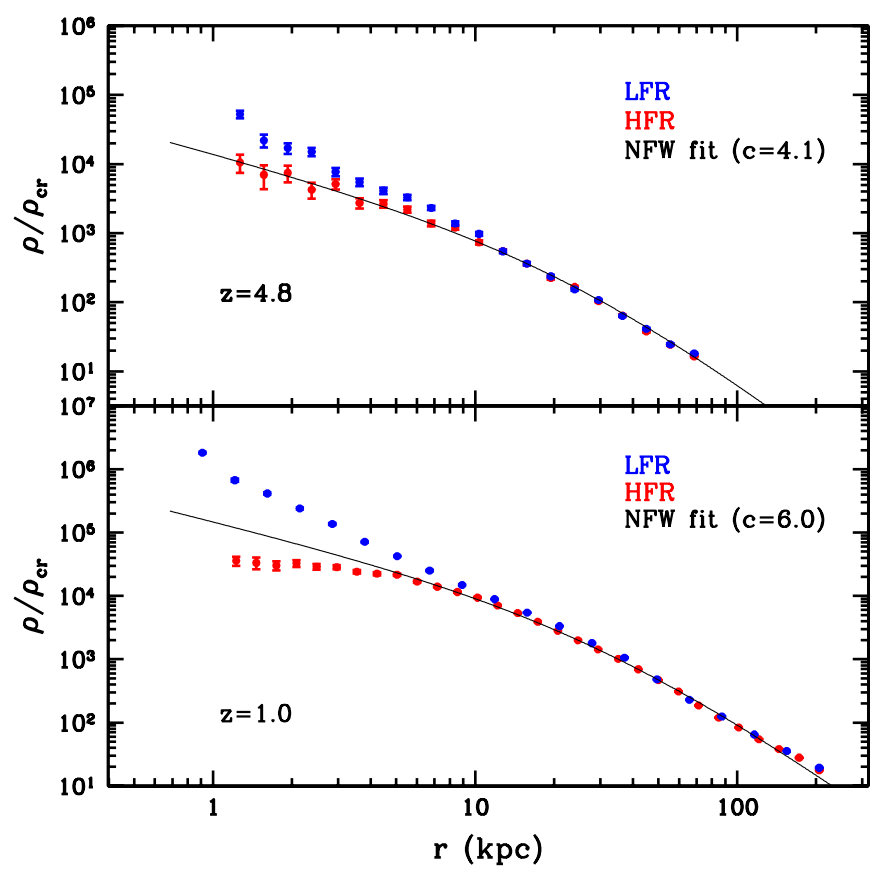

Figure 3. Redshift evolution of the dark matter density profile. In both panels the NFW fit has been obtained by fixing the concentration $c_{\mathrm{vir}}=4.1$ and $c_{\mathrm{vir}}=6.0$ at $z=4.8$ and 1.0, respectively, according to results of Muñoz-Cuartas et al. (2011) based on $N$-body cosmological simulations.

(A color version of this figure is available in the online journal.)

\subsection{When and How Is the Density Profile Flattened?}

Figure 3 shows the dark matter density profile for the hydrodynamical simulations (low and high feedback) at $z=4.8$ and $z=1$ (upper and lower panel, respectively). The two 


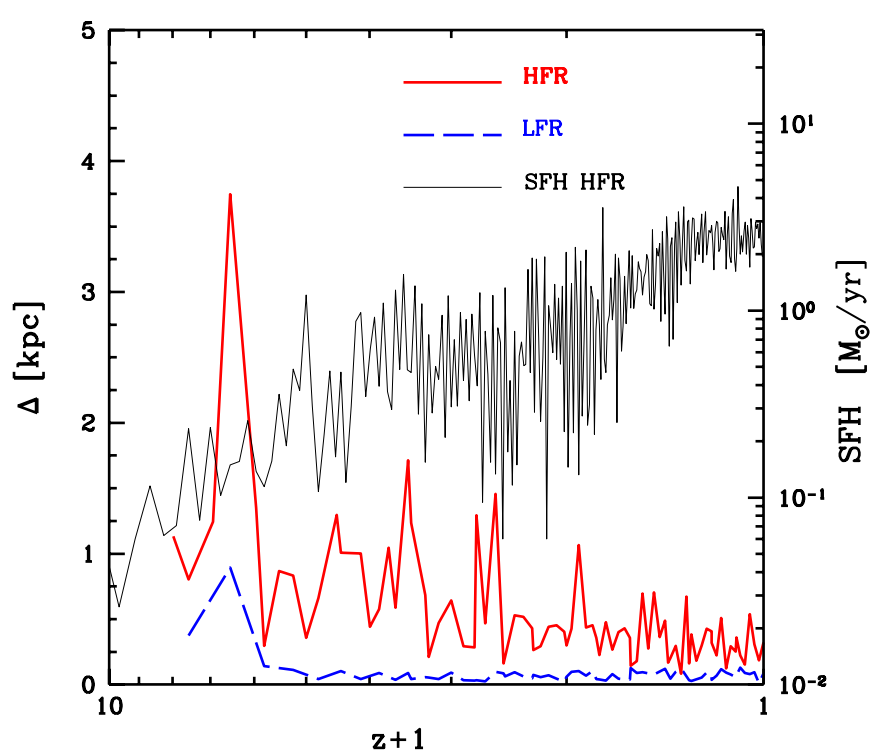

Figure 4. Evolution of the distance between the position of the dark matter and gas potential minima. The solid (red) line and the dashed (blue) line represent the high and low feedback case, respectively. The thin black line shows the star formation history for the high feedback run.

(A color version of this figure is available in the online journal.)

runs show markedly different behaviors: high feedback results in low star formation rates in low-mass progenitors, as it prevents significant gas cooling to the very central regions of the dark matter halos. The dark matter profile remains unperturbed from pure $N$-body simulations (black solid line). In the low feedback case, gas cools rapidly to the central regions at high $z$, and the dark matter adiabatically contracts. At $z=1$ (lower panel), the energy transfer from gas to dark matter in the HFR has already considerably flattened the density profile of this latter component that now clearly deviates from $N$-body-based expectations. The profile of the MUGS run (LFR) is still contracted and has reached a logarithmic slope of $\alpha=2$.

The creation of a core in the dark matter distribution has previously been attributed to rapid variations on the potential due to the bulk motion of gas clouds (Mashchenko et al. 2008; Pontzen \& Governato 2011). In Figure 4 we quantify this variation by plotting the distance, $\Delta$, between the position of the most bound dark matter $\left(\vec{x}_{\mathrm{DM}}\right)$ and gas $\left(\vec{x}_{\text {gas }}\right)$ particles. In the HFR (red line) the rapidly changing potential is reflected in the oscillations of, $\Delta$ with time, with the amplitude of the oscillations of the order of the size of the dark matter core $(\approx \mathrm{kpc})$. In the LFR, the roughly constant and small value of $\Delta$ indicates a more stable potential. This indicates that the changing potential is responsible for generating dark matter cores in our HFR. We note that while bulk gas motions are a natural result of star formation and feedback, it is harder to conceive how such a mechanism would work with active galactic nucleus feedback.

\section{SUMMARY AND CONCLUSIONS}

Several mechanisms have been proposed to flatten dark matter profiles, in order to reconcile the tensions between the observed cored profiles and the cusps of CDM predictions. Fully cosmological simulations have been able to show that such processes can occur in low-mass (dwarf galaxy) systems
(Governato et al. 2010). Yet cosmological simulations of more massive disk galaxies have invariably resulted in adiabatic contraction (Gnedin et al. 2011), contradicting observed disk galaxies (Oh et al. 2008; Donato et al. 2009). The centrally peaked rotation curves and high stellar mass fractions of these simulated galaxies also fail to reproduce observations.

In this work we have, for the first time, explicitly considered the feedback from radiation pressure due to massive stars in a cosmological hydrodynamical simulation of galaxy formation. We compared the dark matter density profile of this new simulation with a twin run that only considered relatively weak feedback from supernovae. We have explicitly shown that stronger stellar feedback can reverse the effect of adiabatic contraction, and expand dark matter halos massive enough to host L* galaxies. The cored profiles in our simulated galaxy have a core radius and a central density that agree with observations (see Figure 2). Simultaneously, the high feedback simulations have rotation curves and stellar masses that are also better matches to observed disk galaxies than their low feedback counterparts.

The flattening of the profile is due to the fluctuation of the global potential, both in its depth (Pontzen \& Governato 2011) and its position (Figure 4). The flattened profile arises at intermediate redshifts, when strong star formation and subsequent energy injection from feedback in shallower potential wells has the strongest effect. At high redshift $(z \approx 3)$ the dark matter density profile is still in agreement with NFW-like predictions (Muñoz-Cuartas et al. 2011).

This pilot study shows that, with reasonable baryonic feedback, there is enough energy input in the central region of the galaxy to induce rapid change in the potential and, eventually, induce a dark matter halo expansion, helping in reconciling observations with CDM predictions. We emphasize that, while the simulation presented here does a good job in reproducing the observed dark matter profiles, it does not addresses all aspects of the galaxy formation.

We are working on expanding our current work to explore the effects of our feedback on a range of properties of galaxies in a wide range of masses (e.g., the two objects already shown in Figure 2). The case of low surface brightness galaxies, in particular, will be interesting since they also present cored density profiles yet have very low baryon fractions. Furthermore, higher resolution simulations will be required to confirm the impact of stellar feedback on the formation of density profiles in galaxies of varying masses. However, we hope this work represents a first step down a new path toward creating more realistic galaxies.

We thank Aaron Dutton for helpful comments on an early version of this manuscript. A.V.M. also thanks P. Salucci and F. Donato for sending an electronic version of their data points and Brent Groves for useful conversations. Numerical simulations were performed on the THEO cluster of the Max-PlanckInstitut für Astronomie at the Rechenzentrum in Garching, on the Universe cluster run by COSMOS in Cambridge, on the SHARCNET clusters in Canada, and on the University of Central Lancashire High Performance Computing Facility. A.V.M. acknowledges funding by Sonderforschungsbereich SFB 881 "The Milky Way System" (subproject A1) of the German Research Foundation (DFG). B.K.G. acknowledges the support of the UK's Science \& Technology Facilities Council (ST/F002432/1 and ST/H00260X/1). 


\section{REFERENCES}

Abadi, M. G., Navarro, J. F., Steinmetz, M., \& Eke, V. R. 2003, ApJ, 597, 21 Agertz, O., Teyssier, R., \& Moore, B. 2011, MNRAS, 410, 1391

Blumenthal, G. R., Faber, S. M., Flores, R., \& Primack, J. R. 1986, ApJ, 301, 27

Brook, C. B., Kawata, D., Gibson, B. K., \& Freeman, K. C. 2004, ApJ, 612, 894 Brook, C. B., Stinson, G., Gibson, B. K., et al. 2011, MNRAS

Burkert, A. 1995, ApJ, 447, L25

Chabrier, G. 2003, PASP, 115, 763

Courteau, S., Dutton, A. A., van den Bosch, F. C., et al. 2007, ApJ, 671, 203

Dekel, A., \& Silk, J. 1986, ApJ, 303, 39

Diemand, J., Zemp, M., Moore, B., Stadel, J., \& Carollo, C. M. 2005, MNRAS, 364,665

Donato, F., Gentile, G., Salucci, P., et al. 2009, MNRAS, 397, 1169

Dutton, A. A., Conroy, C., van den Bosch, F. C., et al. 2011, MNRAS, 416, 322

Dutton, A. A., van den Bosch, F. C., Dekel, A., \& Courteau, S. 2007, ApJ, 654, 27

El-Zant, A., Shlosman, I., \& Hoffman, Y. 2001, ApJ, 560, 636

Gentile, G., Salucci, P., Klein, U., \& Granato, G. L. 2007, MNRAS, 375, 199

Gnedin, O. Y., Ceverino, D., Gnedin, N. Y., et al. 2011, arXiv:1108.5736

Gnedin, O. Y., Kravtsov, A. V., Klypin, A. A., \& Nagai, D. 2004, ApJ, 616, 16

Gnedin, O. Y., Weinberg, D. H., Pizagno, J., Prada, F., \& Rix, H.-W. 2007, ApJ, 671,1115

Governato, F., Brook, C., Mayer, L., et al. 2010, Nature, 463, 203

Governato, F., Willman, B., Mayer, L., et al. 2007, MNRAS, 374, 1479

Guedes, J., Callegari, S., Madau, P., \& Mayer, L. 2011, ApJ, 742, 76

Komatsu, E., Smith, K. M., Dunkley, J., et al. 2011, ApJS, 192, 18

Kroupa, P., Tout, C. A., \& Gilmore, G. 1993, MNRAS, 262, 545

Kuzio de Naray, R., McGaugh, S. S., \& Mihos, J. C. 2009, ApJ, 692, 1321

Macciò, A. V., Dutton, A. A., \& van den Bosch, F. C. 2008, MNRAS, 391, 1940

Macciò, A. V., Moore, B., Stadel, J., \& Diemand, J. 2006, MNRAS, 366, 1529

Mashchenko, S., Couchman, H. M. P., \& Wadsley, J. 2006, Nature, 442, 539
Mashchenko, S., Wadsley, J., \& Couchman, H. M. P. 2008, Science, 319, 174 Mo, H. J., \& Mao, S. 2004, MNRAS, 353, 829

Moster, B. P., Somerville, R. S., Maulbetsch, C., et al. 2010, ApJ, 710, 903

Muñoz-Cuartas, J. C., Macciò, A. V., Gottlöber, S., \& Dutton, A. A. 2011, MNRAS, 411, 584

Navarro, J. F., Eke, V. R., \& Frenk, C. S. 1996, MNRAS, 283, L72

Navarro, J. F., Frenk, C. S., \& White, S. D. M. 1997, ApJ, 490, 493

Navarro, J. F., \& Steinmetz, M. 2000, ApJ, 538, 477

Ogiya, G., \& Mori, M. 2011, ApJ, 736, L2

Oh, S.-H., de Blok, W. J. G., Walter, F., Brinks, E., \& Kennicutt, R. C., Jr. 2008, AJ, 136, 2761

Okamoto, T., Eke, V. R., Frenk, C. S., \& Jenkins, A. 2005, MNRAS, 363 1299

Piontek, F., \& Steinmetz, M. 2011, MNRAS, 410, 2625

Pontzen, A., \& Governato, F. 2011, arXiv:1106.0499

Prada, F., Klypin, A. A., Cuesta, A. J., Betancort-Rijo, J. E., \& Primack, J. 2011, arXiv: 1104.5130

Robertson, B., Yoshida, N., Springel, V., \& Hernquist, L. 2004, ApJ, 606, 32

Salucci, P., \& Burkert, A. 2000, ApJ, 537, L9

Sánchez-Blázquez, P., Courty, S., Gibson, B. K., \& Brook, C. B. 2009, MNRAS, 398,591

Scannapieco, C., White, S. D. M., Springel, V., \& Tissera, P. B. 2009, MNRAS, 396, 696

Shen, S., Wadsley, J., \& Stinson, G. 2010, MNRAS, 407, 1581

Spano, M., Marcelin, M., Amram, P., et al. 2008, MNRAS, 383, 297

Spergel, D. N., Verde, L., Peiris, H. V., et al. 2003, ApJS, 148, 175

Springel, V., Wang, J., Vogelsberger, M., et al. 2008, MNRAS, 391, 1685

Stinson, G., Seth, A., Katz, N., et al. 2006, MNRAS, 373, 1074

Stinson, G. S., Bailin, J., Couchman, H., et al. 2010, MNRAS, 408, 812

Trujillo-Gomez, S., Klypin, A., Primack, J., \& Romanowsky, A. J. 2011, ApJ, 742,16

Wadsley, J. W., Stadel, J., \& Quinn, T. 2004, New Astron., 9, 137

White, S. D. M., \& Rees, M. J. 1978, MNRAS, 183, 341 